\title{
Advances in structure-based drug design of novel bacterial topoisomerase inhibitors
}

\author{
"While models and crystal structures of the gyrase enzyme were known, it was clear that little was truly known about the \\ precise binding mode of the NBTIs...For GlaxoSmithKline, this changed when a crystal structure of a S. aureus \\ DNA gyrase-DNA-NBTI complex was solved, using a multidisciplinary in-house team."
}

\begin{abstract}
Keywords: antibacterial mode of action " bacterial topoisomerase inhibitors $\approx$ novel bacterial topoisomerase inhibitors $\approx$ quinolone antibacterials w treatment of resistant bacterial infection $=\mathrm{x}$-ray structure-based drug design
\end{abstract}

The quinolone class of antibacterial agents, exemplified by ciprofloxacin, represents one of the most important classes of antibacterial agents [1]. Although the targets of these antibacterial agents, bacterial type II topoisomerases (BTs) have been investigated for the past 40 years [2-5], $\mathrm{x}$-ray crystal structures of the quinolone-BT binding mode have only appeared very recently [6-8]. In addition, x-ray structures of a novel class of BT inhibitors that are structurally distinct from the quinolones have recently been published [9]. The novel bacterial topoisomerase inhibitors (NBTIs) and quinolones occupy different binding pockets, providing a structural basis for their lack of cross-resistance to quinolone-resistant strains. The knowledge gained by these recent crystal structures generates unprecedented opportunities for structural optimization and design of new topoisomerase inhibitors.

\section{What was previously known about NBTIs?}

We have been working in the NBTI field for over a decade, our first patent application appearing in 1999 [101]. In 2007, two communications describing a series of nonquinolone pyrazole and tetrahydroindazole inhibitors of type II topoisomerases were published by a group at Johnson and Johnson [10,11]. Although topoisomerase enzymatic activity and antibacterial potencies were given, there was only a brief insight into the mode of action of these inhibitors. The evidence to suggest a novel mode of action for these inhibitors was the absence of DNA cleavage complexes (in contrast to quinolones) and also similar antibacterial potencies against both quinolone-susceptible and -resistant strains of
Staphylococcus aureus. Novel binding to the topoisomerase enzymes is not surprising given the structural differences between quinolones and these NBTIs.

In 2008, a Novexel group published a detailed description of the mode of action of their lead compound NXL-101 [12]. Again, a nonquinolone mode of action was cited due to mutational findings, which show resistance caused by different topoisomerase enzyme mutations to those observed in quinolone-resistant strains. The key mutations conferring quinolone resistance are alterations to Ser-84 of S. aureus GyrA (equivalent to Ser-83 in Escherichia coli GyrA), whereas key mutations conferring NXL-101 resistance are alterations to $S$. aureus GyrA Asp-83. The relative potencies of NXL-101 and representative quinolones (ciprofloxaxin and moxifloxacin) in functional assessments of the topoisomerase II (gyrase) and IV enzymes were also discussed, which again highlighted the differences between quinolones and NBTIs.

In 2009, this Novexel group reviewed the clinical progress of NBTIs, especially Viquidicin (NXL-101), which was discontinued due to QT prolongation in Phase I clinical trials [13]. The paper asserts that this is an extremely active patent field with activity from GlaxoSmithKline, Pfizer, Astra-Zeneca, Aventis, Morphochem and Toyama.

While models and crystal structures of the gyrase enzyme were known, it was clear that little was truly known about the precise binding mode of the NBTIs and, therefore, progress on medicinal chemistry relied on classical structure-activity relationship (SAR) techniques such as pharmacophore modeling and optimization of 'Lipinski-like' properties.

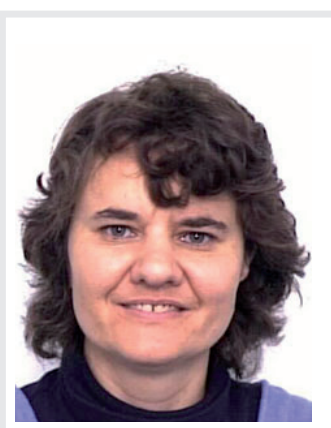

\section{Katherine Widdowson}

Author for correspondence: Antibacterial Discovery Performance Unit, Infectious Diseases CEDD, GlaxoSmithKline, 1250 South Collegeville Road, Collegeville, PA 19426, USA

Tel.: +I 6109176809

E-mail: katherine.l.widdowson@ gsk.com

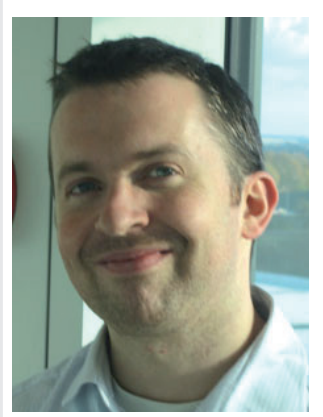

Alan Hennessy

Antibacterial Discovery Performance Unit, Infectious Diseases CEDD, GlaxoSmithKline, Gunnels Wood Road, Stevenage, SGI 2NY, UK

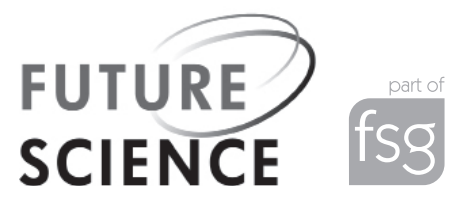


For GlaxoSmithKline, this changed when a crystal structure of a $S$. aureus DNA gyraseDNA-NBTI complex was solved, using a multidisciplinary in-house team [9]. The crystal structures of quinolones and related quinazolinediones inhibitors bound to topoisomerase IV-DNA complexes have now also been published elswehere [6-8].

\section{General comments on the S. aureus DNA gyrase-DNA-NBTI complex}

Prior to solving the crystal structure, it had already been discovered that NBTI GSK299423 had the previously discussed key differences of quinolones: no dsDNA cleavage complexes and lack of cross-resistance to quinolone-resistant bacterial strains (caused by both topoisomerase II [gyrase] and IV mutations). A broad spectrum of activity against multidrug-resistant bacteria was also noted for GSK299423.

The $2.1 \AA$ crystal structure of a complex of an NBTI with DNA and $S$. aureus gyrase also clarified a number of points regarding the mode of action of NBTIs.

Replacement of the DNA-cleaving catalytic S. aureus GyrA Tyr-123 residues with phenylalanine allowed a precleavage complex to be isolated. We believe that the uncleaved DNA is stretched upon complex formation with the gyrase enzyme, but that GSK299423 then arrests the complex prior to the double-strand cleavage. This is a key mechanistic difference from the quinolones as they stabilize the postcleavage DNA-gyrase complex. Crystallographic data show that for a similar DNA-topoisomerase IV-quinolone construct, a quinolone molecule intercalates at each of two DNA-binding sites at the site of DNA cleavage, close to Acinetobacter baumannii ParC Ser-84 (equivalent to Ser-83 in E. coli GyrA), thus explaining the differences in resistance profiles for quinolones versus NBTIs [8].

Although an unprecedented view of the catalytic cycle of the DNA gyrase enzyme is observed, as medicinal chemists, we focused on the key binding regions of our NBTI ligand.

\section{DNA-binding region}

The cyano-quinoline (LHS) unit of GSK299423 intercalates between the two central base pairs of a stretched 20-base pair DNA duplex, midway between the two gyrase active sites on the twofold axis of symmetry.

An important feature for medicinal chemistry has been that the LHS, linker, central unit and oxathiolo-pyridine (RHS) can generally be independently changed and key subunits can therefore be evaluated systematically. To date, over 100 examples of LHS, linker and central units, and over 550 RHS units, have been assessed.

With knowledge from the crystal structure, we examined the LHS-DNA binding interactions. These LHS-DNA interactions have been difficult to measure but we have used computational tools such as ab initio calculations to prioritize new series, especially where arduous syntheses are involved. Detailed analysis showed the accuracy of this system in predicting active and inactive LHS units for a variety of systems.

The twofold symmetry of the binding site led us to scaffolds with alternative LHS linking points, which in turn further drove the SAR of the series.

The search to find additional interactions in the DNA-binding region continues, in order to improve the potency of our novel NBTIs. A variety of LHS systems exists in the NBTI patent literature and more will no doubt appear in the future.

\section{Gyrase protein-binding region}

A noncatalytic pocket appears upon complex formation allowing the RHS unit of GSK299423 to enter and bind via a series of van der Waals interactions with the Ala-68, Gly-72, Met-75 and Met-121 residues.

This pocket is hydrophobic and its size appears to offer limited scope for a large variety of structural change. An additional interaction involves the methylene group flanked by oxygen and sulfur atoms, which appear to form an unusual hydrogen bond with the carbonyl oxygen of Ala-68 (distance of $3.2 \AA$ ) [14]. The observation of this interaction helped clarify some of the SAR observed for this unit. Apart from the RHS unit, aryl-azinone units have also been described as being alternative RHS units of potent NBTIs.

These key residues are largely conserved across Gram-positive and Gram-negative bacteria (both gyrase and Topo IV enzymes), thereby offering us a chance to design dual-targeting, broad-spectrum antibacterials. One difference is the change of Met-121 in S. aureus to Ile or Leu in many Gram-negative species. This modest alteration may offer some opportunities to develop more potent Gram-negative agents, exploiting the slightly larger pocket found in Gram-negative bacteria. 
The size of the pocket does place some restriction on the size of the unit that can bind within, and the patent literature on NBTIs appears largely focused on small bicyclic ring systems.

Just outside this pocket, the basic nitrogen of GSK299423 forms an interaction with the other Asp- 83 residue. We and the Novexel group have shown that mutation of Asp-83, or the nearby in space Met-121 residue, reduces both target and antibacterial potency of NBTIs and so this finding is in excellent correlation with previous data.

This crystal structure provides strong evidence for retaining this interaction. Replacing this amine with a view to reducing $h E R G$ liability may have seemed favorable, but we had more success in keeping this key feature and instead lowering the overall $\log \mathrm{D}$ of our compounds. Indeed, in the NBTI patent literature this basic amine is rarely absent, indicating challenges in finding replacements.

Given that no major additional interactions are present it would appear that the central unit's key role is to ensure the correct positioning of the LHS and RHS units. Using the vectors from the LHS and RHS units it is possible to predict the optimal linker geometry. In our experience an eight-atom link between the LHS and RHS is generally optimal. Many variants have appeared already and the central link provides an opportunity to modulate physicochemical properties without compromising target potency.

\section{Conclusion}

Emerging knowledge of the crystal structures of NBTI and quinolone ligands bound into topoisomerase-DNA complexes has given medicinal chemists an unprecedented opportunity to design novel antibacterials, with a view to overcoming clinical resistance, especially to marketed quinolones.

\section{Bibliography}

1 Mitcher LA. Bacterial topoisomerase inhibitors: quinolone and pyridone antibacterial agents. Chem. Rev. 105(2), 559-592 (2005).

2 Gellert M, Mizuuchi K, O’Dea MH, Nash HA. DNA gyrase: an enzyme that introduces superhelical turns into DNA. Proc. Natl Acad. Sci. USA 73(11), 3872-3876 (1976).

3 Cozzarelli NR. DNA gyrase and the supercoiling of DNA. Science 207(4434), 953-960 (1980).

The future may bring yet more advances in our knowledge of the mechanism of these toposisomerase enzymes and allow de novo design of the next generation of NBTIs. The complex nature of the catalytic cycle permits several points of intervention, in addition to those relevant to the quinolones and NBTIs, which may offer yet more novel antibacterial lead starting points.

There can now be little doubt as to the need for novel mechanism antibacterials to combat the ever-growing threat of resistant bacterial infection and, given the torturous process from hit compound to marketed antibacterial, the time to act is now.

The development of novel quinolones and NBTI antibacterials is now at a pivotal point, history may judge us poorly if we fail.

\section{Acknowledgements}

We thank all GlaskoSmithKline scientists, past and present, who have worked on this program, for their efforts. In particular we wish to acknowledge the two program leaders Neil Pearson and Mick Gwynn.

Financial \& competing interests disclosure

Alan Hennessy was funded by the Wellcome Trust Seeding Drug Discovery Initiative and contract HDTRA1-07-90002 with the US Department of Defence Joint Science and Technology Office for Chemical and Biological Defence and the Defence Threat Reduction Agency's Transformational Medical Technologies. Katherine Widdowson is an employee of GlaxoSmithKline. The views expressed in this article are those of the authors and do not necessarily reflect the official policy or position of the US Department of Defence or the US Government. The authors have no other relevant affiliations or financial involvement with any organization or entity with a financial interest in or financial conflict with the subject matter or materials discussed in the manuscript apart from those disclosed. No writing assistance was utilized in the production of this manuscript.

4 Drevensek P, Kosmrlj J, Giester G et al. X-ray crystallographic, NMR and antimicrobial activity studies of magnesium complexes of fluoroquinolones - racemic ofloxacin and its $S$-form, levofloxacin. J. Inorg. Biochem. 100(11), 1755-1763 (2006).

5 Edwards MJ, Flatman RH, Mitchenall LA et al. A crystal structure of the bifunctional antibiotic simocyclinone D8, bound to DNA gyrase. Science 326(5958), 1415-1418 (2009).

6 Laponogov IS, Sohi MK, Veselkov DA et al. Structural insight into the quinolone-DNA cleavage complex of type IIA topoisomerases. Nat. Struct. Mol. Biol. 16(6), 667-669 (2009).
7 Laponogov I, Pan X, Veselkov DA et al. Structural basis of DNA-gate breakage and resealing by type II topoisomerases. PLoS ONE 5(6), 1-8 (2010).

8 Wohlkonig A, Chan PF, Fosberry AP et al. Structural basis of quinolone inhibition of type IIA topoisomerases and target-mediated resistance. Nat. Struct. Mol. Biol. 17(9), 1152-1153 (2010).

9 Bax BD, Chan PF, Eggleston DS et al. Type IIA topoisomerase inhibition by a new class of antibacterial agents. Nature 466(19), 935-940 (2010). 


\section{EDITORIAL | Widdowson \& Hennessy}

10 Gomez L, Hack MD, Wu J et al. Novel pyrazole derivatives as potent inhibitors of type II topoisomerases. Part 1: synthesis and preliminary SAR analysis. Bioorg. Med. Chem. Lett. 17(10), 2723-2727 (2007).

11 Wiener JJM, Gomez L, Venkatesan $\mathrm{H}$ et al. Tetrahydroindazole inhibitors of bacterial type II topoisomerases. Part 2: SAR development and potency against multidrugresistant strains. Bioorg. Med. Chem. Lett. 17(10), 2718-2722 (2007).
12 Black MT, Stachyra T, Platel D et al. Mechanism of action of the antibiotic NXL101, a novel nonfluoroquinolone inhibitor of bacterial type II topoisomerases Antimicrob. Agents. Chemother. 52(9), 3339-3349 (2008).

13 Black MT, Stachyra T, Platel D et al. New inhibitors of bacterial topoisomerase GyrA/ParC subunits Curr. Opin. Invest. Drugs 10(8), 804-810 (2009).
14 Jeffrey GA, Saenger W. Hydrogen Bonding in Biological Structures. Springer, NY, USA (1991).

\section{- Patent}

101 GlasxoSmithKline Beecham PLC: WO9937635 (1999). 